\title{
Nimotuzumab abrogates acquired radioresistance of KYSE-I50R esophageal cancer cells by inhibiting EGFR signaling and cellular DNA repair
}

This article was published in the following Dove Press journal:

OncoTargets and Therapy

25 February 2015

Number of times this article has been viewed

\begin{abstract}
Hai Liu'
Weifang Yang ${ }^{2}$

Huaping $\mathrm{GaO}^{3}$

Tingting Jiang'

Bengxin $\mathrm{Gu}^{\prime}$

Qinghua Dong ${ }^{4}$

Wenhong $\mathrm{Xu}^{5}$

Shixiu $\mathrm{Wu}^{6}$

Xiaonan Sun'

'Department of Radiation Oncology, Sir Run Run Shaw Hospital, Sir Run Run Shaw Institute of Clinical Medicine of Zhejiang University, Hangzhou, People's Republic of China; ${ }^{2}$ Department of Radiation Oncology, Laboratory of Cellular and Molecular Radiation Oncology, Taizhou Hospital, Wenzhou Medical College, Taizhou, People's Republic of China; ${ }^{3}$ Department of Pharmacy, Sir Run Run Shaw Hospital, School of Medicine, Zhejiang University, Hangzhou, People's Republic of China; ${ }^{4}$ Biomedical Research Center, Sir Run Run Shaw Hospital, School of Medicine, Zhejiang University, Hangzhou, People's Republic of China; ${ }^{5}$ Department of Radiation Oncology, The Second Affiliated Hospital, School of Medicine, Zhejiang University, People's Republic of China; ${ }^{6}$ Department of Radiation Oncology, Hangzhou Cancer Hospital, Wushan District, Hangzhou First People's Hospital, Hangzhou, People's Republic of China
\end{abstract}

Correspondence: Xiaonan Sun Department of Radiation Oncology, Sir Run Run Shaw Hospital, Sir Run Run Shaw Institute of Clinical Medicine of Zhejiang University, 3 Qinchun Road, Hangzhou, Zhejiang 3100I6, People's

Republic of China

Tel +86 57I 86096916

Fax +86 57I 86044817

Email sunxiaonan@hotmail.com
Background: Acquired radioresistance of cancer is common after repeated irradiation and often leads to treatment failure. This study aimed to examine the effects of nimotuzumab on acquired radioresistance in human esophageal carcinoma cells and to investigate its underlying mechanisms.

Methods: The radioresistant human esophageal carcinoma cell line KYSE-150R was generated by using fractionated irradiation. KYSE-150R cells were pretreated with or without nimotuzumab before ionizing radiation. Cell growth and colony formation were measured to quantitate the effects of radiation. The $\gamma-\mathrm{H} 2 \mathrm{AX}$ foci assay was employed to determine cellular DNA-repairing capacity. The phosphorylation of key molecules involved in the epidermal growth factor receptor (EGFR) signaling pathway and cellular DNA repair was measured by Western blot analysis.

Results: Nimotuzumab enhanced radiation-induced inhibition on cell growth and clonogenic survival in KYSE-150R cells. The average number of $\gamma$-H2AX foci increased in the irradiated cells treated with nimotuzumab. Nimotuzumab inhibited phosphorylation of the EGFR and its downstream molecules AKT and ERK. Phosphorylation of the DNA repair-related proteins DNA-PKcs, ATM, and RAD51 was also inhibited by nimotuzumab.

Conclusions: These results indicate that nimotuzumab can inhibit key cancer survival mechanisms, the EGFR signaling pathway, and DNA repair and thereby reverse acquired radioresistance in KYSE-150R cell line.

Keywords: nimotuzumab, esophageal cancer, EGFR, radiation

\section{Introduction}

Esophageal cancer is an aggressive cancer and accounts for the sixth most common cause of cancer-related death worldwide. Most patients are diagnosed with an advanced-stage disease and have a poor prognosis. ${ }^{1}$ After a multimodal regimen that incorporates surgery, chemotherapy, and radiotherapy (RT), the average 5-year overall survival (OS) rate is less than $20 \%{ }^{2}$ Therefore, a better therapeutic strategy is needed to improve OS.

RT is a major treatment for advanced esophageal carcinoma. However, the local recurrence rate at the initial site of esophageal cancer is approximately $44 \%-61 \%$ after primary definitive chemoradiotherapy. ${ }^{3}$ Tumor intrinsic and acquired radioresistance are two major contributors for treatment failure. In particular, acquired radioresistance often occurs after the first irradiation and reirradiation in recurrent esophageal cancer. A recent study has shown that repeated RT is feasible and effective for recurrent esophageal cancer but can cause severe toxicity. ${ }^{4,5}$ Acquired radioresistance is a common phenomenon during fractionated RT, and the involved mechanisms are not fully understood. 
Many factors, including genetics, morphology, structure, receptor expression, and activity in cancer cells, are believed to play important roles for such effects in cancer. Thus, improving tumor radiosensitivity and reducing acquired radioresistance are two major clinical approaches to improve the treatment outcome in primary and recurrent esophageal cancer.

Overexpression of epidermal growth factor receptor (EGFR) has been reported in $60 \%-70 \%$ of esophageal carcinomas. ${ }^{6}$ High EGFR expression is correlated with poor OS and disease-free survival in esophageal cancer patients. ${ }^{7}$ A clinical study has reported that elevated EGFR expression and activity led to tumor resistance to RT in patients. ${ }^{8}$ Preclinical studies have also shown that overactive EGFR induced by irradiation could promote tumor cell proliferation and enhance DNA repair, which might result in tumor radioresistance..$^{9,10}$ Therefore, inhibition of EGFR activity and signaling became an effective strategy to increase tumor radiosensitivity in $\mathrm{RT}$.

Nimotuzumab is a humanized anti-EGFR monoclonal antibody that binds to the extracellular domain of the EGFR and inhibits EGF binding and signaling. ${ }^{11}$ In preclinical studies, nimotuzumab showed antiproliferative, proapoptotic, and antiangiogenic activities in head and neck tumors, which overexpress the EGFRs. ${ }^{12}$ Nimotuzumab has been approved for the treatment of head and neck tumors and glioma in several countries ${ }^{13,14}$ and is under clinical trials for pancreatic cancer, non-small-cell lung cancer, and esophageal cancer. The most exciting part of nimotuzumab use is the lack of severe adverse effects. ${ }^{15}$ For esophageal cancer, nimotuzumab has been found to enhance the radiosensitivity of the EGFR-expressing esophageal squamous cell carcinoma (ESCC) cells in an in vitro study. ${ }^{16}$ Recent clinical studies have demonstrated that nimotuzumab in combination with irradiation was safe and tolerable and yielded encouraging OS, progression-free survival, and local control in ESCC patients. ${ }^{17}$ However, it is still not clear whether nimotuzumab can reverse acquired radioresistance of ESCC. Using a radiation-resistant human esophageal carcinoma cell line, KYSE-150R, we have studied the capacity of nimotuzumab to reverse radiation resistance and involved molecular mechanisms, and demonstrated that nimotuzumab can significantly reduce radioresistance in esophageal cancer cells, suggesting a potential clinical application in RT for esophageal caner.

\section{Methods and materials Cell culture}

Human KYSE-150R cells, which derived from KYSE-150 cells, were gifts from Dr Shi-xiu Wu. ${ }^{18}$ Cells were cultured in RPMI 1640 supplemented with 10\% fetal bovine serum (Gibco, Life Technologies, Carlsbad, CA, USA), $100 \mathrm{U} / \mathrm{mL}$ penicillin, and $100 \mathrm{ug} / \mathrm{mL}$ streptomycin. Cells in the logarithmic phase were used for all experiments.

\section{Cytotoxicity and proliferation assay}

A standard 3-(4,5-dimethylthiazol-2-yl)-2,5-diphenyltetrazolium bromide (MTT) assay was performed to measure the cytotoxicity of nimotuzumab. Cells $\left(4 \times 10^{3}\right.$ cells per well $)$ were cultured in 96-well plates for 24 hours, then incubated with different concentrations of nimotuzumab $(250-4,000 \mathrm{nM})$ and cisplatin $(5-80 \mu \mathrm{M})$ for another 72 hours. Relative absorbance of MTT was measured to calculate cell growth inhibition.

For proliferation assay, cells $\left(2 \times 10^{4}\right.$ cells/well $)$ were seeded in 24 -well plates initially $\left(37^{\circ} \mathrm{C}, 5 \% \mathrm{CO}_{2}\right.$ incubator overnight), then pretreated with nimotuzumab $(1,000 \mathrm{nM})$ for 24 hours before irradiation ( $6 \mathrm{~Gy}$ ). After irradiation, the numbers of living cells were counted daily for 7 days using Trypan Blue staining. The growth curve was done using GraphPad Prism 5.0 software (GraphPad Software Inc., La Jolla, CA, USA).

\section{lonizing radiation}

Irradiation was performed using $6 \mathrm{MV} \mathrm{X}$-rays generated by a linear accelerator at a dose rate of $2 \mathrm{~Gy} / \mathrm{min}$ (PRIMUS-M, Siemens, Erlangen, Germany). Radiation field was $20 \times 20 \mathrm{~cm}$. The source-skin distance was $100 \mathrm{~cm}$.

\section{Colony-forming assay}

Cells cultured in six-well plates were irradiated with X-rays (0-6 Gy) and continuously cultured for another 10-14 days to allow colony formation. Cells were washed with cold phosphate-buffered saline, fixed with $75 \%$ methanol, and stained with $0.5 \%$ crystal violet in methanol. The colonies consisting of $>50$ cells were counted under a dissecting microscope. The surviving fraction was calculated by the GraphPad Prism 5.0 software based on the multitarget/ single-hit model $(\mathrm{SF}=1-[1-\mathrm{e}-\mathrm{D} / \mathrm{D} 0] \mathrm{N})$. The sensitizing enhancement ratio (SER) was calculated based on the formula $\mathrm{SER}=\mathrm{D} 0$ (control cells)/D0 (testing cells). D0 represents the dose that can reduce cell survival to $37 \%$.

\section{Immunoblotting and immunofluorescence staining}

Western blotting was performed as described previously. ${ }^{19}$ For immunofluorescence staining, cells were cultured on a coverslip $(12 \times 12 \mathrm{~mm})$ and irradiated ( $2 \mathrm{~Gy})$. Cells were then fixed and stained with anti- $\gamma-\mathrm{H} 2 \mathrm{AX}$ antibody (Ser139) and 
subsequently with a fluorescein isothiocyanate-conjugated secondary antibody. Cell nuclei were counterstained with 4',6-diamidino-2-phenylindole (Sigma-Aldrich Co., St Louis, MO, USA). The slides were observed and photographed under an LSM710 inverted confocal microscope (Zeiss, Oberkochen, Germany). $\gamma$-H2AX foci in the nuclei were observed and counted. The average number of foci per cell is shown in Figure 3.

\section{Reagents}

Nimotuzumab was provided by Biotech Pharmaceuticals Co., Ltd (Beijing, People's Republic of China). Anti-EGFR, antiphospho-EGFR (S1981), antiphospho-AKT (S473), and anti-AKT and antiphospho-ERK1/2 and anti-ERK1/2 antibodies were purchased from Cell Signaling Technology, Inc. (Danvers, MA, USA). Antiphospho- $\gamma$ H2AX (Ser139), anti-RAD51, anti-ATM, and antiphospho-ATM (Ser1981) and antiglyceraldehyde 3-phosphate dehydrogenase antibodies were purchased from Epitomics, Inc. (Burlingame, CA, USA). As a negative antibody control, rabbit immunoglobulin $\mathrm{G}$ ( $\mathrm{IgG}$ ) was from Hangzhou HuaAn Biotechnology Co. (Hangzhou, People's Republic of China). Antiphospho-DNA-PKcs (T2609) antibody was purchased from Abcam (Cambridge, MA, USA).

\section{Statistical analysis}

All data were presented as mean \pm standard deviation. Statistical significance $(P<0.05)$ was determined by the Student's $t$-test.

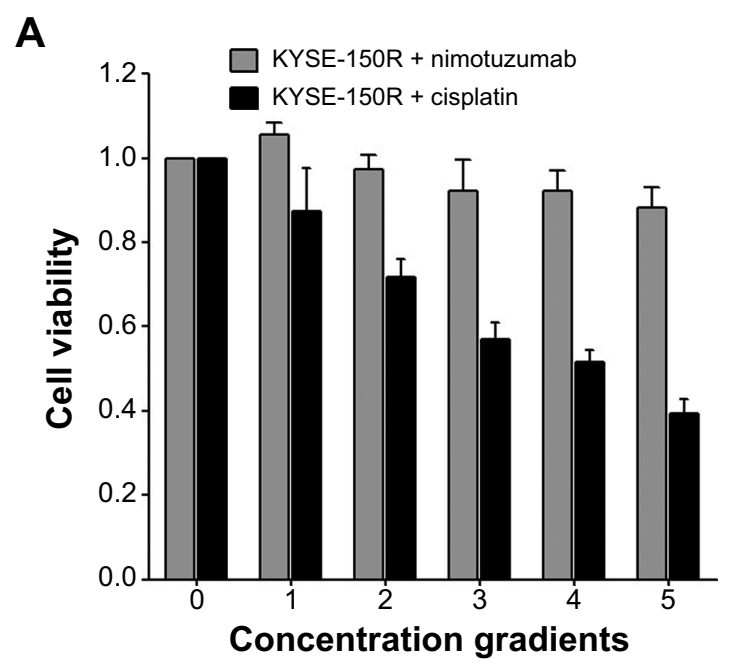

\section{Results \\ Nimotuzumab enhances radiation- induced proliferation inhibition in KYSE-I50R cells}

We first measured the possible toxicity of nimotuzumab and found very limited toxicities of nimotuzumab up to 4,000 nM for 72 hours of incubation in KYSE-150R cells in an MTT assay (Figure 1A). Next, we investigated whether nimotuzumab affects cancer cell proliferation in the presence of irradiation. We treated the cells with or without nimotuzumab (1,000 $\mathrm{nM})$ after ionizing radiation (IR) (6 Gy) and then compared cell proliferation. We used rabbit $\mathrm{IgG}$ as a negative antibody control. Our results showed that the growth rate of two treatment groups was similar within 3 days after IR. However, the cell growth was moderately inhibited in the group treated by nimotuzumab as compared with the mock group at day 4 and day 5 after IR $(P<0.05)$. At day 7 after radiation, cell proliferation was significantly inhibited in both groups but with a greater inhibition in the nimotuzumab-treated group $(P<0.05)$ (Figure 1B).

\section{Nimotuzumab reverses radioresistance in KYSE-I50R cells}

The effects of nimotuzumab on radiosensitivity were measured by clonogenic survival assay, and the results are shown in Figure 2 and Table 1. In Figure 2, KYSE-150R cells are more radioresistant than their parent KYSE150 cells. Pretreatment with nimotuzumab significantly

B

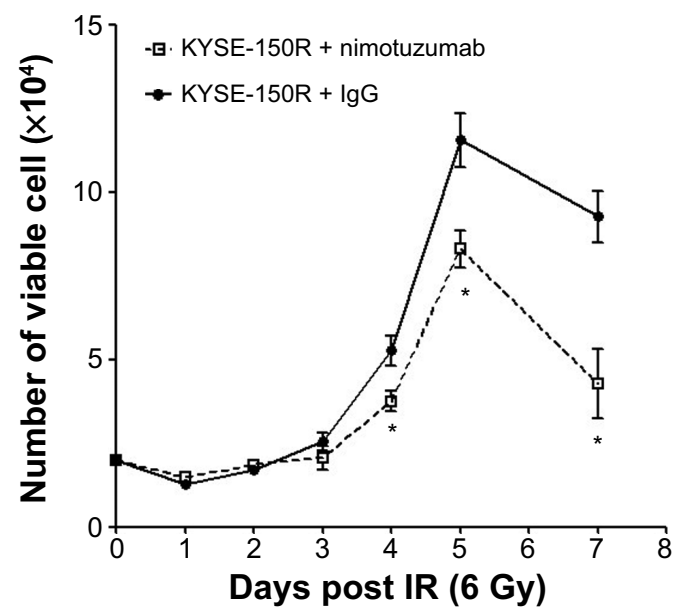

Figure I Effects of nimotuzumab on cell growth in KYSE-I50R cells.

Notes: (A) The cells were treated with different concentrations of nimotuzumab $(250,500,1,000,2,000$, and $4,000 \mathrm{nM})$ for 72 hours. The group was treated with different concentrations of cisplatin $(5,10,20,40$, and $80 \mu \mathrm{M})$ as positive controls. The cell viability was measured in a 3-(4,5-dimethylthiazol-2-yl)-2,5-diphenyltetrazolium bromide assay. (B) Cell proliferation was determined after irradiation (6 Gy) with rabbit lgG or nimotuzumab (I,000 nM). Rabbit lgG serves as a negative antibody control. The data are shown as means \pm standard deviation from three independent experiments. ${ }^{*} P<0.05 \mathrm{KYSE}-\mathrm{I} 50 \mathrm{R}+\operatorname{lgG}$ versus $\mathrm{KYSE}-\mathrm{I50R}+$ nimotuzumab.

Abbreviations: IgG, immunoglobulin G; IR, ionizing radiation. 


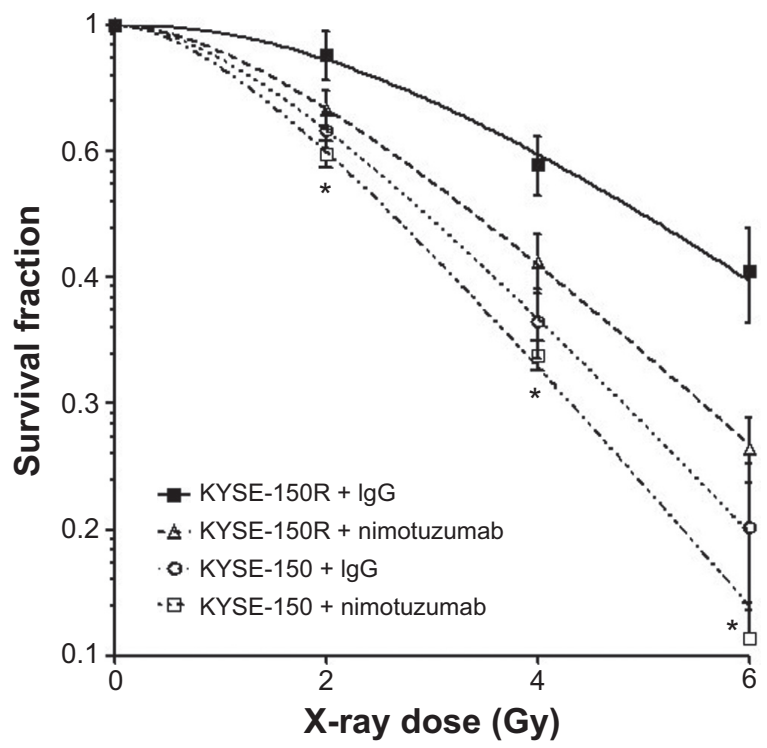

Figure 2 The dose survival curve of KYSE-I50R cells.

Notes: Both KYSE-I50R cells and KYSE-I50 cells (parent cells, radiosensitive) cultured in six-well plates were pretreated with rabbit IgG or nimotuzumab $(I, 000$ $\mathrm{nM}$ ) for 4 hours and followed by IR. Surviving colonies were stained with crystal violet and counted. The survival curves after IR are shown. Data represent mean \pm standard deviation from three independent experiments. $* P<0.05$; KYSE-I50R + IgG versus KYSE-I50R + nimotuzumab.

Abbreviations: IgG, immunoglobulin G; IR, ionizing radiation.

increased radiosensitivity of KYSE-150R cells as compared with IgG treatment. To quantitatively compare their radiosensitivity, we calculated the $\mathrm{D} 0$ values, the doses that can reduce cell survival to $37 \%$. Their D0 values were 3.42 versus 2.75 before and after treatment with nimotuzumab, respectively. The SER was calculated as 1.24. Interestingly, we found that nimotuzumab can also increase radiosensitivity of the parental KYSE-150 cell. However, the radiationsensitizing effect of nimotuzumab on KYSE-150 cells is weaker than on KYSE-150R cells, comparing SER (1.09 in KYSE-150 vs 1.24 in KYSE-150R). Because KYSE-150R cells, which were generated by fractionated radiation, are acquired radioresistant cells, we can conclude that nimotuzumab treatment may reverse acquired radiation resistance of esophageal cancer cells.

Table I The parameters of a multitarget model based on clonogenic survival assay

\begin{tabular}{llllll}
\hline Cell line & N & D0 & Dq & SF2 & SER \\
\hline KYSE-I5OR + IgG & 2.637 & 3.42 & 3.32 & 0.89 & \\
KYSE-I5OR + nimo & 2.024 & 2.75 & 1.94 & 0.73 & 1.24 \\
KYSE-I50 + IgG & 2.022 & 2.38 & 1.68 & 0.68 & \\
KYSE-I50 + nimo & 1.96 & 2.17 & 1.46 & 0.63 & 1.099 \\
\hline
\end{tabular}

Abbreviations: $\mathrm{N}$, extrapolation number that measures the width of shoulder of the survival curve; D0, the dose that reduces cell survival to $37 \%$; Dq, the intercept of the extrapolated high dose; SF2, surviving fraction at 2 Gy; SER, sensitivity enhancement ratio; lgG, immunoglobulin G; nimo, nimotuzumab.

\section{Nimotuzumab reduces DNA repair after irradiation in KYSE-I50R cells}

We further assessed whether nimotuzumab affects cellular DNA repair after irradiation using the $\gamma-\mathrm{H} 2 \mathrm{AX}$ foci assay. The $\gamma-\mathrm{H} 2 \mathrm{AX}$ foci number reflects unrepaired double-strand breaks (DSBs) in an irradiated cell. The average number of $\gamma-\mathrm{H} 2 \mathrm{AX}$ foci per cell was counted during 1-48 hours after a 2 Gy irradiation (Figure 3). There was no difference in the average numbers of $\gamma$-H2AX foci at 4 hours after IR between the KYSE-150R cells treated by the combined nimotuzumab/radiation and the cells treated with radiation/IgG. However, a significantly greater number of $\gamma-\mathrm{H} 2 \mathrm{AX}$ foci were observed between 8 hours and 48 hours after IR in the nimotuzumab-treated group as compared with the IgG-treated group $(P<0.05)$. These results indicate that nimotuzumab can inhibit cancer cell DNA repair, showing more $\gamma$-H2AX foci in KYSE-150R cells.

\section{Nimotuzumab inhibits radiation-induced activation of EGFR signaling pathway in KYSE-I50R cells}

To investigate how nimotuzumab enhances cancer cell radiosensitivity, we first compared the expression levels and the activities of EGFR in KYSE-150 and KYSE-150R cells. Although the same expression levels of EGFR were detected in both cells, radiation induced a significantly higher level of phosphorylation in KYSE-150R cells, as compared with the level in KSYE-150 cells (Figure 4A and Supplementary Figure S1). Moreover, we monitored the phosphorylation levels of EGFR and its downstream signaling molecules, including AKT and ERK, after pretreatment with nimotuzumab followed by irradiation. Radiation activated EGFR, AKT, and ERK in KYSE-150R cells, as expected. Pretreatment with nimotuzumab inhibited not only radiation-induced phosphorylation of EGFR but phosphorylation of the downstream molecules AKT and ERK. The effects of nimotuzumab peaked between 30 minutes and 2 hours post-IR (6 Gy) (Figure 4B and Supplementary Figure S1). These data demonstrated the inhibitory properties of nimotuzumab on the EGFR signaling pathway in radioresistant KYSE-150R cells.

\section{Nimotuzumab diminishes the radiation- induced phosphorylation of DNA-PKcs and ATM, and downregulates the expression levels of RAD5 I in KYSE-I50R cells}

DNA DSBs are repaired by two major mechanisms: the homologous recombination (HR) and the nonhomologous 


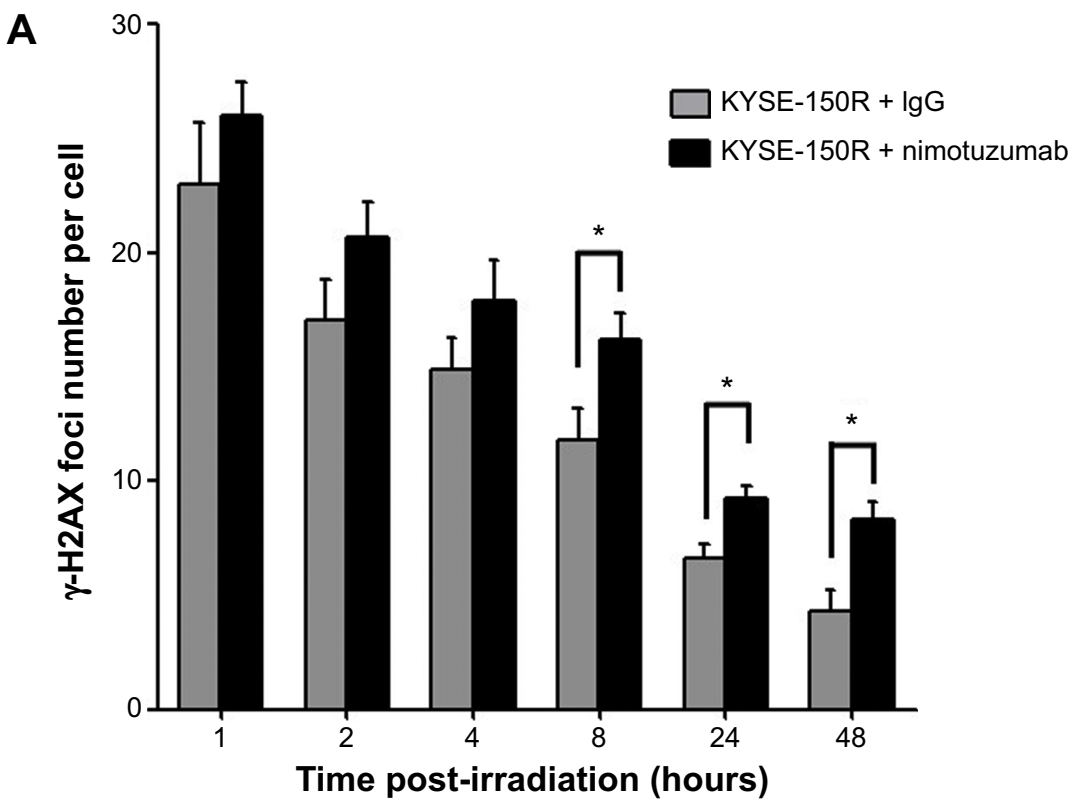

B

Figure 3 The average number of $\gamma-\mathrm{H} 2 \mathrm{AX}$ foci per cell in KYSE-I50R

Notes: Nimotuzumab increases the number of $\gamma$-H2AX foci. KYSE-I50R cells were pretreated with rabbit lgG or nimotuzumab (I,000 nM) for 24 hours, followed by 2 Gy of irradiation. (A) The average number of $\gamma-\mathrm{H} 2 \mathrm{AX}$ foci per cell was counted and is shown. Each bar represents mean \pm standard deviation. At least $\mathrm{I} 00$ nuclei were evaluated per sample. (B) The representative nuclei with $\gamma-\mathrm{H} 2 \mathrm{AX}$ foci under an immunofluorescent microscope. *Indicates statistical significance $(P<0.05)$.

Abbreviations: IgG, immunoglobulin G; DAPI, 4',6-diamidino-2-phenylindole.

end joining (NHEJ) in mammalian cells. DNA-PKcs and ATM coordinately regulate DSB repair through the aforementioned mechanisms. To examine whether nimotuzumab interferes with these molecules in DNA repair, KYSE-150R cells were treated as described previously. Phosphorylation of ATM and DNA-PKcs was examined and is shown in Figure 5A. The basal levels of autophosphorylation of ATM and DNA-PKcs were very low in KYSE-150R cells. Radiation induced significant phosphorylation of ATM and DNA-PKcs, which peaked at 2 hours after irradiation. Pretreatment with nimotuzumab significantly attenuated such effects (Figure 5A and Supplementary Figure S2). In addition, we measured the expression levels of RAD51, a central component of HR. Interestingly, the expression levels of RAD51 were increased by radiation but attenuated by nimotuzumab in a time-dependent manner (Figure 5B and Supplementary Figure S2). These results suggest that nimotuzumab may inhibit DNA repair by inhibiting RAD51 expression.

\section{Discussion}

Acquired radioresistance plays a crucial role in RT failure. To conquer this clinical obstacle, we studied this clinically important problem in a radiation-resistant cancer cell line (KYSE-150R), which was generated by fractionated radiation, a commonly used method in cancer RT for its advantages in preventing normal tissues. As compared with the previous report on the effects of cetuximab, we further focused on the EGFR signaling pathway and DNA repair in terms of neutralization of the EGFR in esophageal cancer cells. Here, we found a differential pattern of the EGFR activities in response to irradiation in the radioresistant KYSE-150R cells as compared with the radiosensitive KYSE-150. Pretreatment with nimotuzumab significantly decreased phosphorylation of EGFR and its downstream molecules AKT and ERK, the key proteins for cancer survival. Moreover, pretreatment with nimotuzumab inhibited DNA repair after irradiation by modulating the activities of DNA-PKcs, ATM, and RAD51.

The radiation-induced activation of EGFR and its downstream signaling cascades is linked to radiation protection through promoting cell proliferation and DNA repair. ${ }^{10}$ It has been reported by Schmidt-Ullrich et $\mathrm{al}^{20}$ in 1994 that increased expression of EGFR was seen in MCF-7 cells after repeated radiation exposure. We now report that greater levels of EGFR phosphorylation were observed in the radioresistant KYSE-150R cells after irradiation. We did not find significant changes in the expression levels of EGFR in KYSE-150R cells. We believe that chronic and fractionated radiation accounts for such effects. Selective survival of radioresistant cells and/or induction of gene mutations may ultimately lead to acquired radioresistance. ${ }^{21}$ 
A

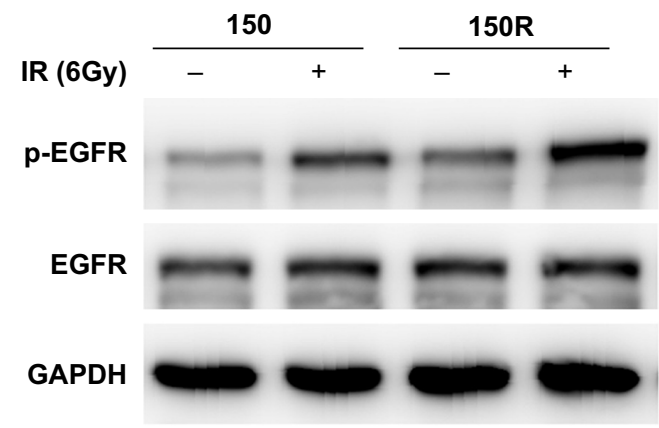

B

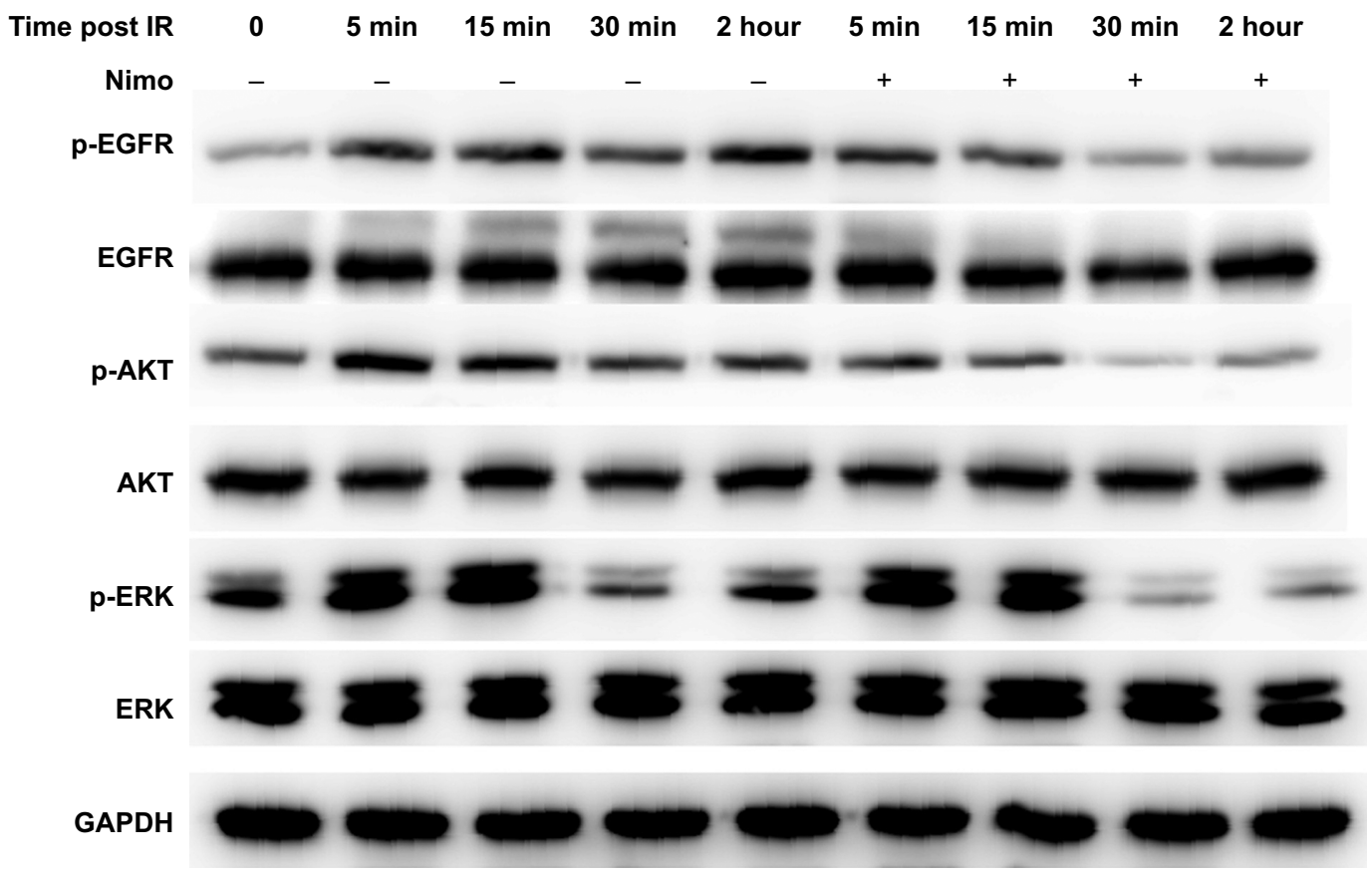

Figure 4 Nimotuzumab inhibits radiation-induced activation of EGFR signaling pathway in KYSE-I50R cells.

Notes: (A) Radiation-induced phosphorylation of EGFR in KYSE-I50 and KYSE-I50R cells. Total cell lysate was harvested 2 hours after exposure to radiation (6 Gy) and used to measure the levels of phosphorylated EGFR, total EGFR, and GAPDH. (B) Nimotuzumab suppresses the radiation-induced activation of EGFR and its downstream signaling molecules in KYSE-I50R cells. Cells were pretreated with nimotuzumab (I,000 nM for 24 hours) and then irradiated. Total cell lysate was harvested at indicated time points after irradiation for Western blot analysis. Total EGFR, p-EGFR, total AKT, p-AKT, total ERK, and p-ERK were detected in Western blotting. GAPDH served as a loading control. The quantitative data for the Western blotting results are shown in "Supplementary material".

Abbreviations: EGFR, epidermal growth factor receptor; GAPDH, glyceraldehyde 3-phosphate dehydrogenase; p-EGFR, phosphorylated EGFR; p-AKT, phosphorylated AKT; p-ERK, phosphorylated ERK; IR, ionizing radiation.

It is known that radiation-induced phosphorylation of the EGFR activates the Ras/Raf/ERK(MAPK) and the PI3K-Akt signaling pathways, which can protect cells from radiation-induced cell death but promote cell survival and proliferation. ${ }^{9}$ This is the case in KYSE-150R cells. We further found that pretreatment with nimotuzumab inhibited EGFR as well as ERK and AKT. Taken together, our study confirmed the important role of the EGFR signaling pathway in radioresistance of esophageal cancer cells. We further demonstrated that acquired radioresistance in cancer cells may be reversed by manipulation of the EGFR signaling pathway using nimotuzumab.
Radiation kills tumor cells by inducing DNA DSBs, and DSBs can be repaired primarily through two major mechanisms (HR and NHEJ) in mammalian cells. The important roles of DNA-PKcs and ATM in cellular DNA repair have been well documented. ${ }^{22}$ Nimotuzumab inhibited cancer cell DNA repair via its inhibition on DNA-PKcs and ATM, and thereby enhanced the radiosensitivity of KYSE-150R cells. Previous studies have demonstrated that blockade of EGFR signaling can inhibit cellular DNA repair. Golding et a ${ }^{23}$ found that blocking EGFR signaling impaired the formation of both p-(T2609) DNA-PKcs and p-(S1981) ATM foci. 


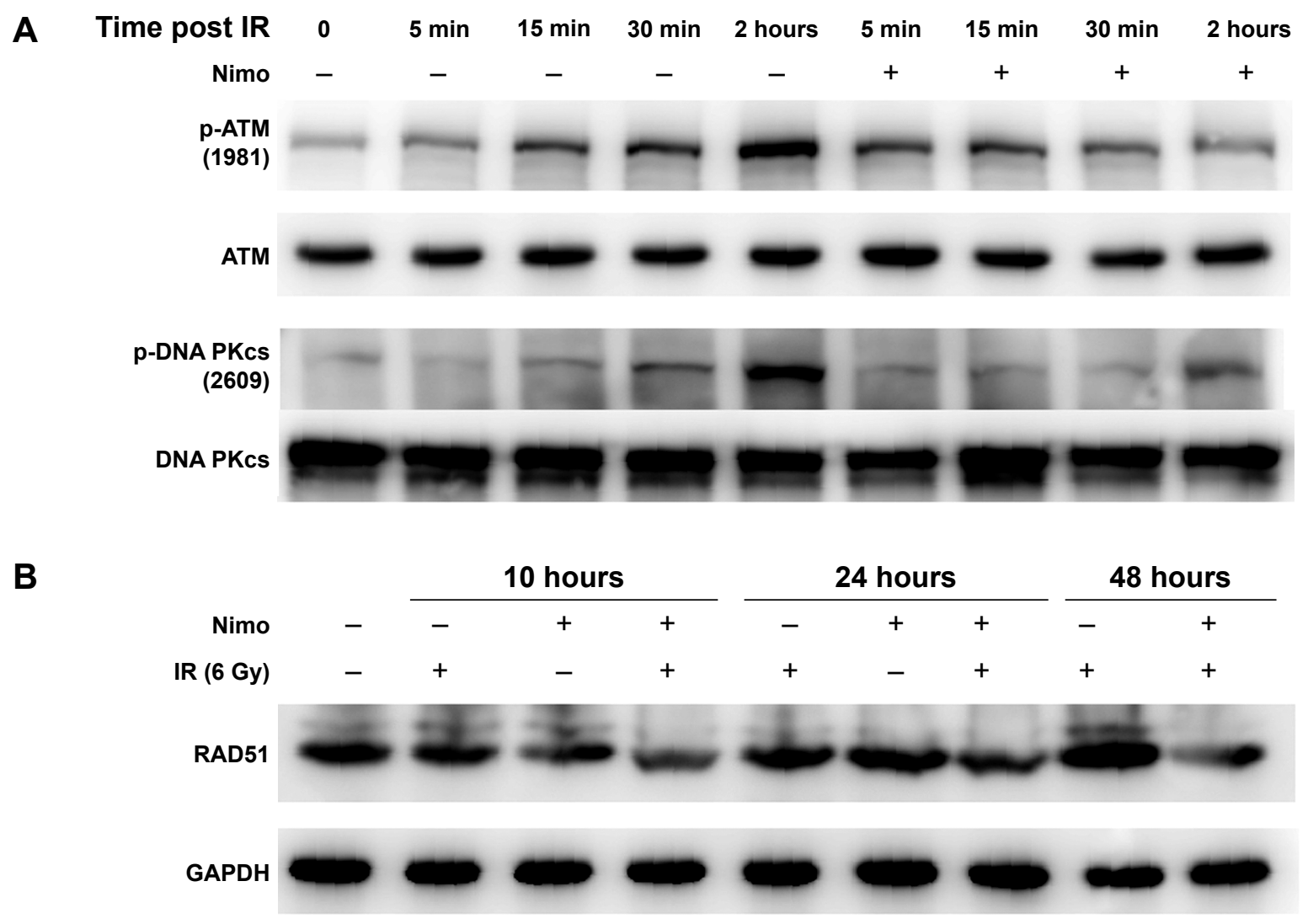

Figure 5 Nimotuzumab inhibits phosphorylation of DNA repair-related proteins in KYSE-I50R cells.

Notes: Nimotuzumab modulates the key molecules in DNA repair, including DNA-PKcs (A), ATM (A), and RAD5I (B). KYSE-I50R cells were preincubated with nimotuzumab $(1,000 \mathrm{nM})$ for 24 hours, followed by irradiation (6 Gy), as indicated. Total cell lysate was collected at the indicated time points after irradiation. GAPDH served as a loading control. The quantitative data for the Western blotting results are shown in "Supplementary material".

Abbreviations: DNA, deoxyribonucleic acid; GAPDH, glyceraldehyde 3-phosphate dehydrogenase.

The translocated EGFR can bind to DNA-PKcs in the nuclei after irradiation and regulate DNA-PKcs phosphorylation. Cetuximab, another neutralizing antibody against the EGFR, can inhibit radiation-induced nuclear translocation of the EGFR, block the interaction between the EGFR and DNAPKcs, and delay cellular DNA repair. ${ }^{24}$ In addition, AKT can also translocate to the nucleus and interact with DNA-PKcs and regulate its activity in irradiated cells. ${ }^{25} \mathrm{~A}$ recent report revealed that the AKT/DNA-PKcs pathway also contributes to acquired radioresistance in tumor cells. ${ }^{26}$

ATM plays a critical role in regulating HR but not NHEJ. ${ }^{27}$ The EGFR signaling can modulate ATM activity through different mechanisms. Golding et $\mathrm{al}^{28}$ found that ERK kinases activity was required for ATM phosphorylation. Blockade of the MEK/ERK signaling compromised ATM kinase activity for efficient $\mathrm{HR}$ in response to radiation. In addition, ATM is required for the correct posttranslational modification of Rad51 and protein complex formation following IR. ${ }^{29}$ Rad51 is important for DSB repair through $\mathrm{HR},{ }^{29}$ and inhibition of Rad51 increases radiosensitivity. ${ }^{30}$
Li et al reported that erlotinib, a selective EGFR inhibitor, inhibited Rad51 expression. ${ }^{31}$ Several studies have shown that overexpression of Rad51 was commonly seen in esophageal cancer tissues, which was correlated with low efficacy of treatment and decreased survival in ESCC patients. ${ }^{32,33}$ Due to their biological function and unique expression pattern, our study and others strongly suggest that ATM and Rad51 are two therapeutic targets to reverse radioresistance and to increase radiosensitivity in cancer therapy.

Clinical trials have already shown that nimotuzumab is a safe and effective biological agent with minor side effects. ${ }^{15}$ In our in vitro radioresistant KYSE-150R cell system, pretreatment with nimotuzumab reversed acquired radioresistance and enhanced radiosensitivity in esophageal cancer cells by inhibiting the EGFR/Akt/DNA-PKcs signaling pathway and reducing ATM and Rad51 activities. Thus, nimotuzumab may be an effective sensitizer for radioresistant malignancies like esophageal cancer. Although further investigations are needed to fully clarify the biological function of nimotuzumab in esophageal cancer, our study 
strongly suggests its clinical applications in RT for recurrent radioresistant cancer.

\section{Acknowledgments}

This work was supported in part by grants from the Chinese National Natural Science Foundation (No 81272494 and 81441086), Natural Science Foundation of Zhejiang Province (No LY14H160016), Major Science and Technology Program of Zhejiang Province (No 2013C03044-6), and Medical and Health Research Program of Zhejiang Province (No 2013KYA115).

\section{Disclosure}

The authors report no conflicts of interest in this work.

\section{References}

1. Jemal A, Bray F, Center MM, et al. Global cancer statistics. CA Cancer J Clin. 2011;61(2):69-90.

2. Zhang Y. Epidemiology of esophageal cancer. World J Gastroenterol. 2013;19(34):5598-5606.

3. Herskovic A, Martz K, al-Sarraf M, et al. Combined chemotherapy and radiotherapy compared with radiotherapy alone in patients with cancer of the esophagus. N Engl J Med. 1992;326(24):1593-1598.

4. Fakhrian K, Gamisch N, Schuster T, et al. Salvage radiotherapy in patients with recurrent esophageal carcinoma. Strahlenther Onkol. 2012;188(2):136-142.

5. Kim YS, Lee CG, Kim KH, et al. Re-irradiation of recurrent esophageal cancer after primary definitive radiotherapy. Radiat Oncol J. 2012;30(4):182-188.

6. Hanawa M, Suzuki S, Dobashi Y, et al. EGFR protein overexpression and gene amplification in squamous cell carcinomas of the esophagus. Int $J$ Cancer. 2006;118(5):1173-1180.

7. Wang KL, Wu TT, Choi IS, et al. Expression of epidermal growth factor receptor in esophageal and esophagogastric junction adenocarcinomas: association with poor outcome. Cancer. 2007;109(4):658-667.

8. Ang KK, Berkey BA, Tu X, et al. Impact of epidermal growth factor receptor expression on survival and pattern of relapse in patients with advanced head and neck carcinoma. Cancer Res. 2002;62(24):7350-7356.

9. Liang K, Ang KK, Milas L, Hunter N, Fan Z. The epidermal growth factor receptor mediates radioresistance. Int J Radiat Oncol Biol Phys. 2003;57(1):246-254.

10. Chen DJ, Nirodi CS. The epidermal growth factor receptor: a role in repair of radiation-induced DNA damage. Clin Cancer Res. 2007;13(22 Pt 1):6555-6560.

11. Mateo C, Moreno E, Amour K, et al. Humanization of a mouse monoclonal antibody that blocks the epidermal growth factor receptor: recovery of antagonistic activity. Immunotechnology. 1997;3(1):71-81.

12. Crombet-Ramos T, Rak J, Perez R, Viloria-Petit A. Antiproliferative, antiangiogenic and proapoptotic activity of h-R3: a humanized antiEGFR antibody. Int $J$ Cancer. 2002;101(6):567-575.

13. Crombet T, Osorio M, Cruz T, et al. Use of the humanized anti-epidermal growth factor receptor monoclonal antibody h-R3 in combination with radiotherapy in the treatment of locally advanced head and neck cancer patients. J Clin Oncol. 2004;22(9):1646-1654.

14. Ramos TC, Figueredo J, Catala M, et al. Treatment of high-grade glioma patients with the humanized anti-epidermal growth factor receptor (EGFR) antibody h-R3: report from a phase I/II trial. Cancer Biol Ther. 2006;5(4):375-379.
15. Boland WK, Bebb G. Nimotuzumab: a novel anti-EGFR monoclonal antibody that retains anti-EGFR activity while minimizing skin toxicity. Expert Opin Biol Ther. 2009;9(9):1199-1206.

16. Zhao L, He LR, Xi M, et al. Nimotuzumab promotes radiosensitivity of EGFR-overexpression esophageal squamous cell carcinoma cells by upregulating IGFBP-3. J Transl Med. 2012;10:249

17. Ma NY, Cai XW, Fu XL, et al. Safety and efficacy of nimotuzumab in combination with radiotherapy for patients with squamous cell carcinoma of the esophagus. Int J Clin Oncol. 2014;19(2):297-302.

18. Jing Z, Gong L, Xie CY, et al. Reverse resistance to radiation in KYSE-150R esophageal carcinoma cell after epidermal growth factor receptor signal pathway inhibition by cetuximab. Radiother Oncol. 2009;93(3):468-473.

19. Liu H, Sun X, Zhang S, et al. The dominant negative mutant Artemis enhances tumor cell radiosensitivity. Radiother Oncol. 2011;101(1):66-72.

20. Schmidt-Ullrich RK, Valerie KC, Chan W, McWilliams D. Altered expression of epidermal growth factor receptor and estrogen receptor in MCF-7 cells after single and repeated radiation exposures. Int J Radiat Oncol Biol Phys. 1994;29(4):813-819.

21. Windholz F. Problems of acquired radioresistance of cancer: adaptation of tumor cells. Radiology. 1947;48(4):398-404.

22. Shrivastav M, Miller CA, De Haro LP, et al. DNA-PKes and ATM co-regulate DNA double-strand break repair. DNA Repair (Amst). 2009;8(8):920-929.

23. Golding SE, Morgan RN, Adams BR, et al. Pro-survival AKT and ERK signaling from EGFR and mutant EGFRvIII enhances DNA double-strand break repair in human glioma cells. Cancer Biol Ther. 2009;8(8):730-738.

24. Dittmann K, Mayer C, Fehrenbacher B, et al. Radiation-induced epidermal growth factor receptor nuclear import is linked to activation of DNAdependent protein kinase. J Biol Chem. 2005;280(35):31182-31189.

25. Toulany M, Kehlbach R, Florczak U, et al. Targeting of AKT1 enhances radiation toxicity of human tumor cells by inhibiting DNAPKcs-dependent DNA double-strand break repair. Mol Cancer Ther. 2008;7(7):1772-1781.

26. Shimura T. Acquired radioresistance of cancer and the AKT/GSK3beta/ cyclin D1 overexpression cycle. J Radiat Res. 2011;52(5):539-544.

27. Golding SE, Rosenberg E, Khalil A, et al. Double strand break repair by homologous recombination is regulated by cell cycleindependent signaling via ATM in human glioma cells. $J$ Biol Chem. 2004;279(15):15402-15410.

28. Golding SE, Rosenberg E, Neill S, et al. Extracellular signal-related kinase positively regulates ataxia telangiectasia mutated, homologous recombination repair, and the DNA damage response. Cancer Res. 2007;67(3):1046-1053.

29. Chen G, Yuan SS, Liu W, et al. Radiation-induced assembly of Rad51 and Rad52 recombination complex requires ATM and c-Abl. J Biol Chem. 1999;274(18):12748-12752.

30. Ohnishi T, Taki T, Hiraga S, Arita N, Morita T. In vitro and in vivo potentiation of radiosensitivity of malignant gliomas by antisense inhibition of the RAD51 gene. Biochem Biophys Res Commun. 1998;245(2):319-324.

31. Li L, Wang H, Yang ES, Arteaga CL, Xia F. Erlotinib attenuates homologous recombinational repair of chromosomal breaks in human breast cancer cells. Cancer Res. 2008;68(22):9141-9146.

32. Li Y, Yu H, Luo RZ, et al. Elevated expression of Rad51 is correlated with decreased survival in resectable esophageal squamous cell carcinoma. J Surg Oncol. 2011;104(6):617-622.

33. Nakanoko T, Saeki H, Morita M, et al. Rad51 expression is a useful predictive factor for the efficacy of neoadjuvant chemoradiotherapy in squamous cell carcinoma of the esophagus. Ann Surg Oncol. 2014 21(2):597-604. 


\section{Supplementary materials}
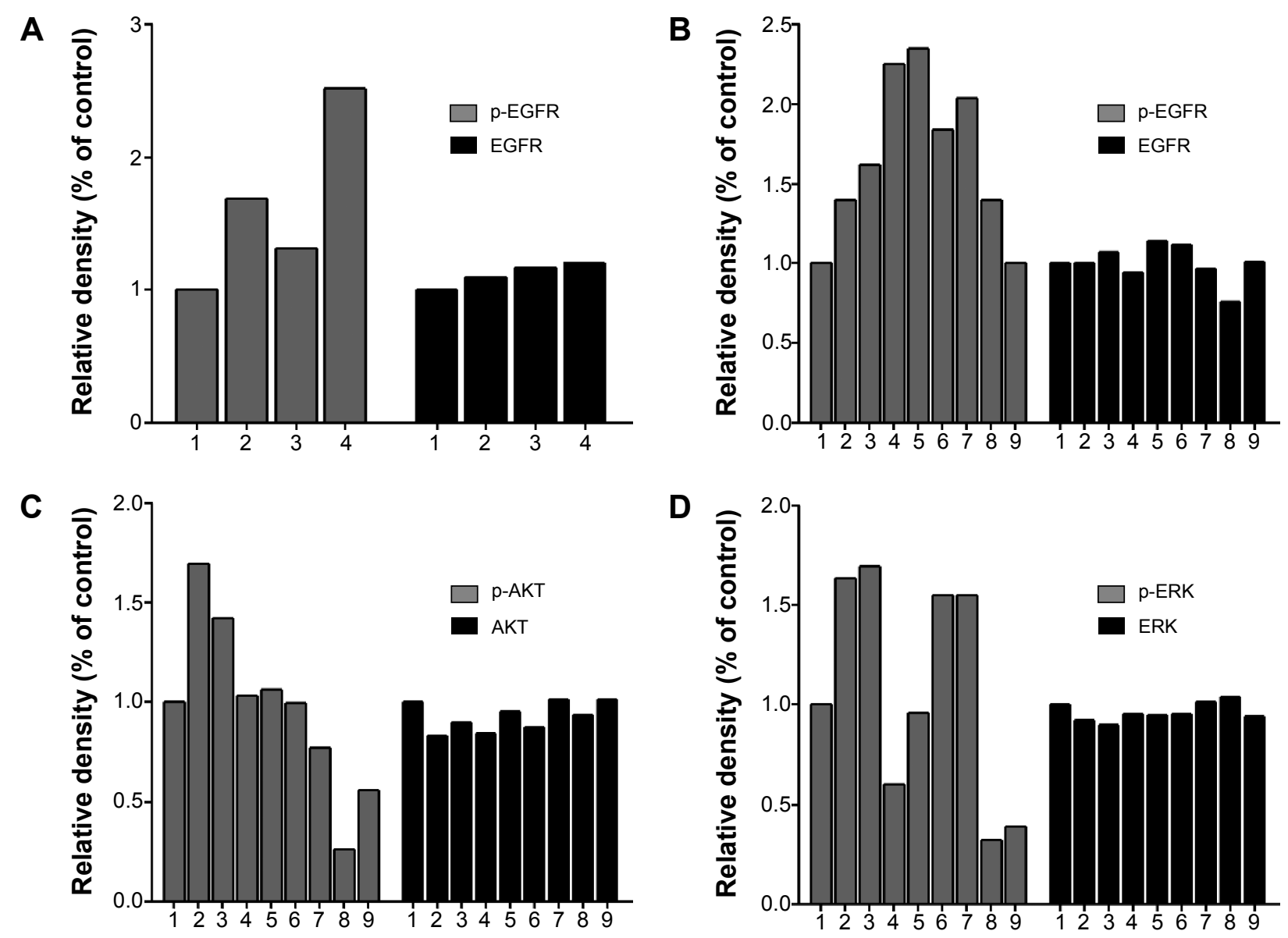

Figure SI The quantitative data for the Western blotting results of Figure 4.

Notes: The quantitative analysis was performed by Quantity One software. The relative densities of the bands were determined and are represented by the rectangular columns, compared with the control group (lane I). The numbers of horizontal ordinate represent the lane numbers from left to right in Figure 4 . (A) The quantitative data for the western blotting results of Figure 4A. (B-D) The quantitative data for the western blotting results of Figure 4B.

Abbreviations: EGFR, epidermal growth factor receptor; p-EGFR, phosphorylated EGFR; p-AKT, phosphorylated AKT; p-ERK, phosphorylated ERK. 
A

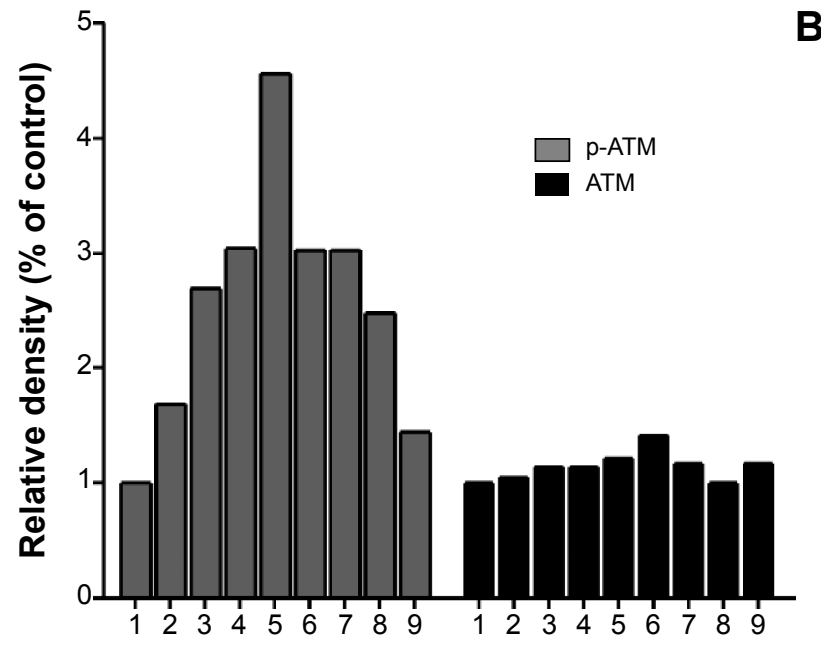

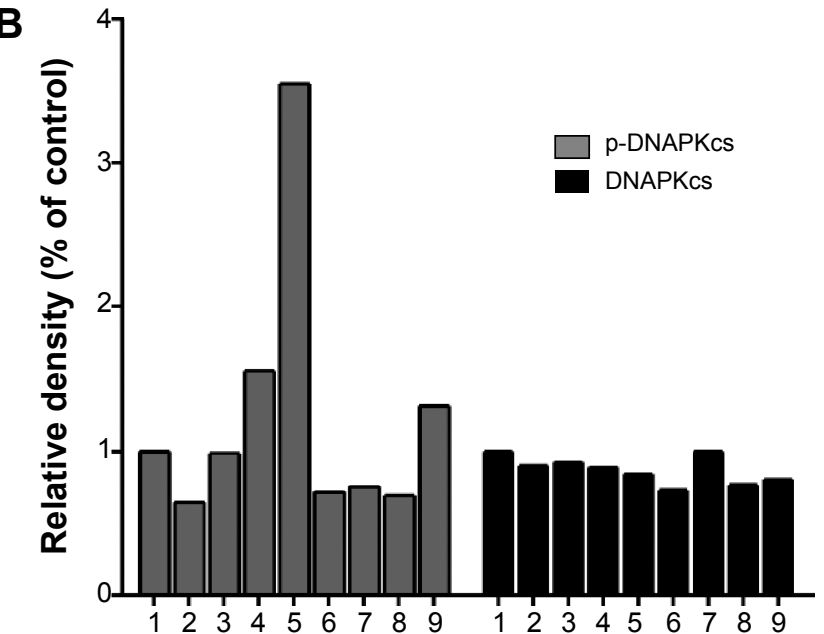

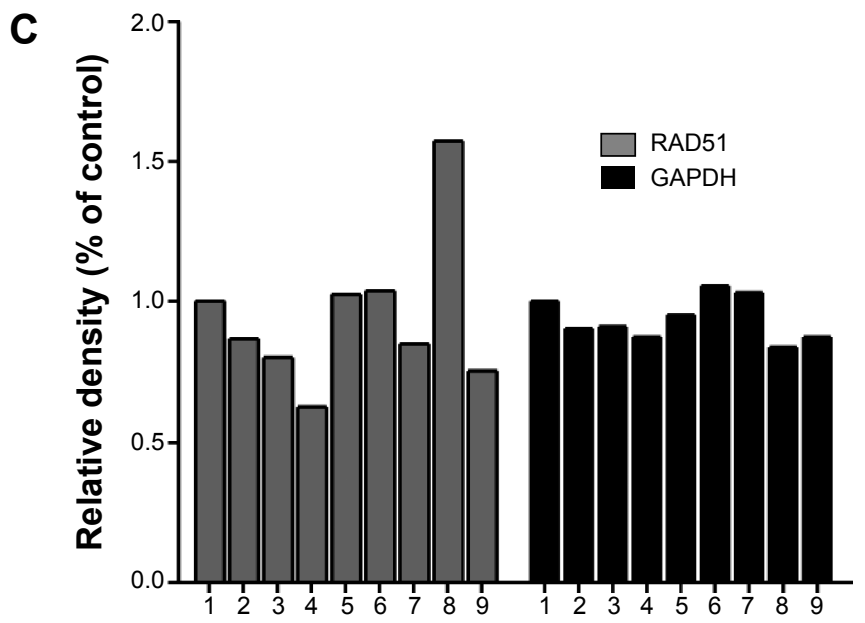

Figure S2 The quantitative data for the Western blotting results of Figure 5.

Notes: The quantitative analysis was performed by Quantity One software. The relative densities of the bands were determined and are represented by the rectangular columns, compared with the control group (lane I). The numbers of horizontal ordinate represent the lane numbers from left to right in figure 5. (A, B) The quantitative data for the western blotting results of Figure 5A. (C) The quantitative data for the western blotting results of Figure 5B.

Abbreviations: pATM, phosphorylated ATM; GAPDH, glyceraldehyde 3-phosphate dehydrogenase; p-DNA PKcs, phosphorylated DNA PKcs.

\section{Publish your work in this journal}

OncoTargets and Therapy is an international, peer-reviewed, open access journal focusing on the pathological basis of all cancers, potential targets for therapy and treatment protocols employed to improve the management of cancer patients. The journal also focuses on the impact of management programs and new therapeutic agents and protocols on patient perspectives such as quality of life, adherence and satisfaction. The manuscript management system is completely online and includes a very quick and fair peer-review system, which is all easy to use. Visit http://www.dovepress.com/testimonials.php to read real quotes from published authors. 